\title{
AlphaHGA; A New Antiviral Substance Against HIV Affecting
} Capsid Assembly

\author{
Anders Vahlne ${ }^{* \ddagger 1,6}$, Samir Abdurahman ${ }^{1}$, Michael Levi ${ }^{6}$, Elin Andersson ${ }^{2}$, \\ Bo Svennerholm² ${ }^{2}$, Peter Horal ${ }^{2}$, Alenca Jejcic ${ }^{1}$, Stefan Höglund ${ }^{3}$, \\ Akos Vegvaris ${ }^{3}$, Marita Högberg ${ }^{4}$ and Jan Balzarini ${ }^{5}$
}

Address: ${ }^{1}$ Division of Clinical Virology, Karolinska Institutet, Stockholm, Sweden, ${ }^{2}$ Department of Medical Microbiology, University of Göteborg Sweden, ${ }^{3}$ Department of Biochemistry, University of Uppsala, ${ }^{4}$ Chemilia AB, Huddinge, Sweden, ${ }^{5}$ Rega Institute, University of Leuven, Belgium and ${ }^{6}$ Tripep AB, Huddinge, Sweden

Email: Anders Vahlne* - anders.vahlne@labmed.ki.se

* Corresponding author $¥$ Presenting author

from 2005 International Meeting of The Institute of Human Virology Baltimore, USA, 29 August - 2 September 2005

Published: 8 December 2005

Retrovirology 2005, 2(Suppl I):S94 doi:10.1 I86/1742-4690-2-SI-S94

\section{Background}

We have previously shown that the tripeptide glycyl-prolyl-glycine-amide (GPG-amide) inhibits HIV-1 replication in vitro by affecting proper capsid assembly of HIV-1. However, GPG-amide failed in a phase II clinical trial on HIV-infected individuals. In the search for what went wrong we have now found that the tripeptide in itself does not exert the antiviral activity but is metabolised in two steps into the active compound by serum enzymes. The first step is cleavage of GPG-amide into GP and glycineamide (G-amide) by the soluble di-peptidyl peptidase CD26. In the present study we show that G-amide is further metabolised to the actve antiviral substance by an enzyme present in foetel bovine serum but not in human serum.

\section{Material and Methods}

Numerous methods have been employed including molecular biology, magnetic resonance (NMR).

\section{Results}

The second step is an enzyme mediated oxidation of Gamide into the active anti-viral compound. By NMR the molecule was found to be alpha-hydroxy glycine amide (alphaHGA). The latter is a small molecule with a molecular mass of 90. The conversion of G-amide into alphaHGA does not take place in human or rodent serum but in the serum from most species including fetal calf and pig serum. Hence, GPG-amide does not affect HIV-1 replication if the infected cells are cultured in the presence of human serum only. We have now synthesized alphaHGA and been able show that the synthesized substance inhibits HIV-1 replication in the presence of human serum only or with heat inactivated fetal calf serum. In the presence of alphaHGA in the culture medium progeny HIV-1 particles have abnormal capsid structures and are non-infectious.

\section{Conclusion}

AlphaHGA is a new promising antiretroviral substance. All preclinical studies on alphaHGA have now been performed and clinical studies on HIV infected individuals are planned to start this year. 